\title{
EuroEcho-Imaging 2013: highlights
}

\section{Patrizio Lancellotti ${ }^{1 *}$, Julien Magne ${ }^{2}$, Bernard Cosyns ${ }^{3}$, Erwan Donal ${ }^{4}$, Anastasia Kitsiou ${ }^{5}$, Owen Miller ${ }^{6}$, Steffen E. Petersen ${ }^{7}$, and Gilbert Habib ${ }^{8}$}

\begin{abstract}
${ }^{1}$ Department of Cardiology, University of Liège Hospital, GIGA Cardiovascular Sciences, Heart Valve Clinic, CHU Sart-Tilman, Liège, Belgium; ${ }^{2} \mathrm{CHU}$ Limoges, Hôpital Dupuytren, Pôle Coeur-Poumon-Rein, Service Cardiologie, Limoges, France; ${ }^{3}$ UZ Brussel- CVHZ, ICMI 101 Laarbeeklaan 1090 Brussels, Belgium; ${ }^{4} \mathrm{CIC}-\mathrm{IT}$ U 804 , CHU Rennes, Université Rennes 1 , Service de Cardiologie, CHU RENNES, France; ${ }^{5}$ Cardiology Department, Sismanoglio Hospital, Athens, Greece; ${ }^{6}$ Department of Congenital Heart Disease, Evelina London Children's Hospital, London, UK; ${ }^{7}$ William Harvey Research Institute, NIHR Cardiovascular Biomedical Research Unit at Barts, Queen Mary University of London, UK; and ${ }^{8}$ Department of Cardiology, Aix-Marseille Université, 13284, Marseille, France. La Timone Hospital, Bd Jean Moulin, 13005, Marseille, France
\end{abstract}

Received 13 January 2014; accepted after revision 16 January 2014; online publish-ahead-of-print 25 February 2014

The annual meeting of the European Association of Echocardiography (EuroEcho-Imaging) was held in Istanbul, Turkey. In the present paper, we present a summary of the 'Highlights' session.

Keywords Heart failure $\bullet$ 3D echocardiography $\bullet$ Cardiac computed tomography $\bullet$ Nuclear cardiology $\bullet$ Cardiovascular magnetic resonance • Congenital heart diseases • Heart valve diseases

\section{Introduction}

The second annual scientific meeting of the European Association of Cardiovascular Imaging (EACVI), EuroEcho-Imaging 2013, was held in Istanbul, Turkey. The meeting was marked by two major events: (i) the celebration of a decade of success of our association in the field of CVI and (ii) the ratification of the new constitution of the unified EACVI structure that integrates the main non-invasive CVI stakeholders. The main themes were heart failure and imaging in interventional cardiology. The meeting was a great success with a record number of participants (3234 delegates) and over 1400 abstracts submitted. The last day of the meeting, the 'Highlights' session wrapped up the event with a summary of the most relevant abstracts presented throughout the congress. A short report of this session is presented below.

\section{Heart failure}

Muraru et al. assessed the mitral valve morphology of 32 patients with hypertrophic cardiomyopathy (HCM) using a semi-automated mitral valve analysis by $3 \mathrm{D}$-transthoracic echocardiography (3D-TTE). ${ }^{1}$ When compared with controls, patients with HCM had significant larger mitral leaflet and annulus areas and greater posterior leaflet/ mitral annulus area ratio. 3D-TTE posterior leaflet area $\left(>4.64 \mathrm{~cm}^{2}\right)$ enabled an excellent discrimination of patients with HCM, and was correlated with $\mathrm{LV}$ remodelling and dynamic obstruction. Takotsubo cardiomyopathy (TTC) is an important differential diagnosis of acute coronary syndrome. ${ }^{2}$ Serial 2D speckle tracking echocardiography was performed in 40 patients with typical pattern of TTC. At admission, a circular impairment in regional strain was reported. Of note, functional recovery was asymmetrical at Day 5 and complete at 1-month follow-up. In LV non-compaction cardiomyopathy (NCC), heart failure is the most common clinical presentation and the main predictor of outcome. ${ }^{3}$ Mansencal et al. evaluated the relationship between the degree and extent of LV NCC and regional myocardial function using 2D speckle tracking imaging. Thirty patients with NCC were compared with 30 patients with dilated cardiomyopathy (DCM). NCC was present in 48 segments (10\%), mainly localized in the apical region and the lateral wall. Borderline C was observed in 80 (16.6\%) segments and normal C was present in $330(68.7 \%)$ segments. Akinesia (19.3\%) and hypokinesia (68\%) were more frequent in $\mathrm{C}$ segments than in NC or borderline segments. Basal and median strain values were similar in both groups. Apical strain values were higher in NCC than that in DCM with a significant apical/basal strain gradient $(P<0.05)$. Many of the life-saving cancer therapies can have significant and serious cardiovascular side effects. During the meeting, several reports focused on the late cardiac effects of anticancer treatments. In 80 patients treated with chemotherapy between 1995 and 2011, Roussin et al. compared standard evaluation of LV systolic function (LV ejection fraction) to 2D speckle tracking myocardial deformation (global longitudinal strain, GLS). ${ }^{4}$ GLS was lower $(-13.9 \pm 3.6$ vs. $-19.2 \pm 1.3 \%$, $P<0.01)$ in cancer survivors than in controls despite preserved LV ejection fraction. ${ }^{4}$ Therefore, GLS analysis is enabled to accurately identify subclinical LV dysfunction. The long-term clinical and prognostic significance of these findings requires further investigation.

* Corresponding author. Tel: + 32436671 94; Fax: + 32436671 95, Email: plancellotti@chu.ulg.ac.be

Published on behalf of the European Society of Cardiology. All rights reserved. (C) The Author 2014. For permissions please email: journals.permissions@oup.com 
In the setting of LV dyssynchrony, although echocardiography has gained progressive interest, its clinical usefulness remains controversial. $^{5-7}$ In heart failure patients eligible for cardiac resynchronization therapy (CRT), predicting the occurrence of ventricular arrhythmias is of importance. Hasselberg et al. studied 73 patients receiving CRT with 2D speckle tracking imaging. Ten (14\%) of these patients developed sustained ventricular tachycardia/ventricular fibrillation during follow-up. While LV ejection fraction was not a marker of ventricular arrhythmia, GLS was. Indeed, LV ejection was similar between groups whereas GLS was more reduced in patients with arrhythmia. The assessment of GLS in heart failure candidate for CRT may help identify those who may benefit from an implantable cardioverter defibrillator. In heart failure, the presence of mitral regurgitation (MR) plays a central role in determining pulmonary flow overload and pulmonary hypertension. ${ }^{8}$ The determinants of right ventricle (RV) adaptation during exercise was assessed in 44 patients with heart failure and preserved LV ejection fraction. MR severity was strongly related to the functional response of the $R V$ during maximal exercise and to ventilatory inefficiency (steeper $\mathrm{VE} / \mathrm{VCO}_{2}$ slope). Right ventricular (RV) dysfunction and failure basically affect the clinical course in heart failure syndrome. In 459 patients with systolic heart failure, Bandera et al. showed that a $<0.36$ TAPSE/PASP ratio (tricuspid annular systolic excursion/pulmonary arterial systolic pressure) could serve as to identify a subset of heart failure patients at higher risk. ${ }^{9}$

\section{Valvular heart disease}

The outstanding role of multi-imaging modalities in the management and risk stratification of patients with valvular heart disease continues to gain interest. The vast majority of abstracts submitted under the topic of valvular heart disease investigated aortic stenosis (AS). In this regard, Tsang et al. reported the outcome results of 106 medically managed patients with symptomatic AS and not suitable for aortic valve replacement (AVR). ${ }^{10}$ As expected, they found a high mortality rate of $56 \%$ during a mean follow-up of 1.8 years. Using various multivariable models, they identified that the best echocardiographic predictors of mortality were LV ejection fraction, four-chamber GLS, mitral E/e' ratio and LA volume. In addition, although the Society of Thoracic Surgeons score was an excellent predictor of mortality, the combination of echocardiographic parameters was statistically better. A specifically dedicated oral session on LV myocardial strain in patients with AS was held during the meeting. Carstensen et al. compared the LV geometry and function of three groups of AS: asymptomatic patients with preserved LV ejection fraction $(n=105$, control group), symptomatic patients with preserved LV ejection fraction $(n=72)$, and symptomatic patients with depressed LV ejection fraction $(n=69) .{ }^{11}$ Of interest, when compared with control group, patients with impaired LV ejection fraction exhibit significantly lower LV myocardial longitudinal, circumferential, and radial strains. However, despite similar LV geometry, mass, and LV circumferential and radial strains, patients with preserved $L V$ ejection fraction had a significant lower LV longitudinal strain than the control group. This was particularly highlighted in LV basal segments. Similarly, in 55 asymptomatic patients with moderate to severe AS and preserved LV ejection fraction, Dulgheru et al. reported that maximal exercise capacity was mainly determined by basal LV myocardial longitudinal strain on multivariable analysis. ${ }^{12}$ In contrast, both mid- and apical LV longitudinal strains were not significantly associated with peak oxygen uptake. Using exercise stress echocardiography, Federspiel et al. have shown that exercise-induced changes in mean transaortic pressure were highly predictive of combined cardiovascular event (only including death and hospitalization for worsening heart failure) in 195 asymptomatic patients with preserved LV ejection fraction and moderate or above AS. ${ }^{13}$ Other predictors of events were an LA surface of $>25 \mathrm{~cm}^{2}$ and previous myocardial infarction. The so-called paradoxical low-flow low-gradient (LFLG) entity of severe AS was extensively discussed during the meeting. Fabris et al. studied a population of 170 severe AS with a mean pressure gradient of $\leq 30 \mathrm{mmHg}$ and LV ejection fraction of $\geq 55 \%$. ${ }^{14}$ These patients depicted the typical pattern of 'paradoxical' LFLG severe AS, i.e. small LV cavity, high degree of LV concentric hypertrophy, and high valvulo-arterial impedance. Furthermore, severe symptoms (NYHA $\geq \mathrm{III}$ ), atrial fibrillation, and no AVR during the follow-up were the main determinants of mortality. In a retrospective multicentre study, Sato et al. have identified LV global longitudinal strain $<17 \%$ as a major predictor of cardiovascular event in 'paradoxical' LFLG AS. ${ }^{15}$ Following transcatheter aortic valve implantation (TAVI), all patients included in the study of Knebel et al. exhibited significantly better LV longitudinal function than before intervention. ${ }^{16}$ Nevertheless, this improvement was more pronounced in 'paradoxical' LFLF severe AS group. Left atrial (LA) function plays a crucial role in patients with MR. The peak systolic longitudinal strain of the LA was quantified using 2D speckle tracking in 276 patients. Cardiovascular events were found in 108 patients and peak atrial longitudinal strain $<15 \%$ was associated with markedly reduced event-free survival. ${ }^{17}$ Kozdag et al. aimed to identify the impact of the presence of tricuspid regurgitation (TR) on 30-day readmission for heart failure. ${ }^{18}$ They studied a large cohort of 706 patients hospitalized for worsening of HF. A moderate to severe TR was reported in $58 \%$ of the 131 patients with 30 -day re-admission for heart failure. In multivariable analysis, TR proved to be a significant independent predictor of early readmission in patients with heart failure.

\section{3-Dimensionnal echocardiography}

Using 2D echocardiography, the proximal isovelocity surface area (PISA) is assumed to be hemispherical. The calculated flow rate across the leaking mitral valve (Qmr) varies with the selected aliasing velocity. In addition, the optimal range of velocities is shifted upward with increasing Qmr. Brugger et al. sought to demonstrate that Qmr derived from 3D PISA was independent of the Nyquist limit, because the PISA surface was directly measured. In patients with MR referred for transoesophageal echocardiography (TOE), the PISA with clear aliasing boundaries were extracted as slices after $3 \mathrm{D}$ reconstruction using customized software. They showed that using Nyquist limits of 30.8 and $41.1 \mathrm{~cm} / \mathrm{s}$, Qmr as measured by 3D PISA was stable; this stability was not influenced by the amount of Qmr. ${ }^{19}$ Accurate assessment of prosthetic valve internal diameter (ID) is essential for effective and safe treatment. Condemi et al. performed 2332 2D/ 3D TOE in vitro measurements on 53 bioprosthetic valves. 2D Echo ID measurements were made in two orthogonal planes at the level of the sewing ring and similar 3D measurements were generated from multi-plane reconstructions. They were compared with both 
the manufacturer supplied valve ID (M.ID) and the True ID (T.ID) measured with Hegar dilators. The authors showed that T.ID was significantly smaller than M.ID, and this might lead to a wrong choice of implanted valve. ${ }^{20}$ Veronesi et al. developed novel custom software to trace and measure tricuspid valve annulus (TVA). When compared with controls, TVA was larger in patients with pulmonary hypertension. The tricuspid annulus was also more circular and with less longitudinal displacement. Changes in annular remodelling were not uniform. The anterior and posterior segments were enlarged, while the septal remained unchanged. This novel software revealed that pulmonary hypertension affects size, shape, and function of the TVA, suggesting that 3D analysis may be useful for evaluation of different RV disease states. ${ }^{21}$ Brugger et al. compared the regurgitant volume (RVol) in 37 patients with chronic MR using TOE and cardiac magnetic resonance (CMR) imaging. For each patient, all PISA visible during systole were 3D reconstructed using customized software. 3D-PISA total RVol was directly related to CMR-RVol. Patients with severe MR according to CMR-RVol $\geq 60 \mathrm{~mL}$ were all correctly classified as severe by $3 \mathrm{D}-\mathrm{PISA}{ }^{22}$ Muraru et al. evaluated the reference values for $3 D$ LV volumes and function parameters comparing vendor with a vendor-independent DICOM-based software in 235 healthy volunteers. Despite the differences in LV parameters obtained with both software were statistically significant, Bland-Altman analysis showed a clinically irrelevant bias and reasonable limits of agreement for all measurements with both software. ${ }^{23}$ Shivalkar et al. studied 146 consecutive patients with an indication for dobutamine stress echocardiography (DSE) with 2D echo, 3Dtriplane and full volume acquisitions with and without myocardial contrast (MCE) given at rest and peak stress for LV opacification. They showed that agreement between $2 \mathrm{D}$ and $3 \mathrm{D}$ stress echo was poor for abnormal vs. normal stress tests. Furthermore, they were able to demonstrate that early revascularization as well as major cardiovascular events at 36 months of follow-up were predicted accurately by 3D-DSE but not by 2D-DSE. ${ }^{24}$ 3D TOE allows a comprehensive evaluation of aortic atheroma. Caminati et al. aimed to develop an automated method to allow reconstruction of the descending aorta from contiguous 3D TOE images for total plaque burden quantification. Algorithm performance was first tested on phantom images. The results on the validation datasets confirmed the feasibility and accuracy of the proposed method and its potential clinical applicability, with increased field-of-view compared with multiple single acquisitions and improved spatial resolution in the overlapped areas. $^{25}$ Debonnaire et al. assessed the characteristics, determinants, and relation between secondary MR severity and mitral valve leaflet remodelling (enlargement) in 24 patients with grade 1-2 MR, 30 patients with grade 3-4 MR, and 22 control subjects with normal structural and functional MV. They demonstrated that the degree and extent of MV leaflet remodelling was independently associated with MR severity. ${ }^{26}$

\section{CMR imaging}

CMR imaging remains the clinical reference for right ventricular volume and function assessment. The analysis of $R V$ volumes requires expertise. Grapsa et al. demonstrated that after the analysis of 120 scans CMR and 3D echocardiography have comparable and excellent inter- and intra-observer agreement, but that the learning curve is better for CMR, likely due to the clearer endocardial border definition. $^{27}$

The CMR response to echo speckle tracking is called tissue or feature tracking. Importantly, this technique, unlike tagging, does not require additional image acquisition, as standard cine images can be used. Orwat et al. demonstrated that myocardial strain measured by CMR related to functional status (NYHA class, $P=0.0005$ ), objective exercise capacity (peak oxygen uptake, $P=0.02$ ), and neurohormonal levels (NT-pro-BNP, $P=0.007$ ) in adults with congenitally corrected transposition of the great arteries independently of global systemic strain measured by echo and CMR LV ejection fraction. ${ }^{28}$

Franzoso et al. compared left and right atrial size and function in arterial switch and atrial switch repair for transposition of the great arteries using CMR. ${ }^{29}$ The atrial size and function in patients after the atrial switch procedure is abnormal compared with patients after the arterial repair and healthy controls. The atrial reservoir and pump function are reduced, the conduit function, however, was increased. Impaired atrial function may be an important determinant of RV failure. Left atrial volumes measured by $2 \mathrm{D}$ echocardiography and CMR were compared by Axelsson et al. in patients with $\mathrm{HCM}{ }^{30}$ There is a systematic underestimation of $L A$ volumes with many $2 \mathrm{D}$ echocardiography methods compared with CMR in healthy controls which, interestingly, was not observed in HCM and the authors speculated whether this was related to different LA geometry in HCM. CMR is also an elegant technique to diagnose myocarditis noninvasively through oedema-sensitive and contrast-based techniques demonstrating myocardial damage different to the one seen following myocardial infarction. Halmai et al. demonstrated reduced systolic deformation indices (3D speckle tracking) in those segments corresponding to myocardial involvement on CMR despite preserved LV ejection fraction. ${ }^{31} 3 \mathrm{D}$ speckle tracking may thus be an alternative technique to identify abnormalities due to myocarditis if CMR is not feasible. Marques et al. found a strong relationship between the extent of late gadolinium enhancement and NT-proBNP $(r=0.74$, $P=0.003$ ) despite mostly preserved LV ejection fraction and concluded that this underlines the role of CMR for providing an in vivo histology. ${ }^{32}$

A prospective cohort study presented by Poyhonen et al. ${ }^{33}$ suggested that the extent of late gadolinium enhancement and segmental wall motion abnormalities on CMR could predict adverse outcomes in non-ischaemic cardiomyopathy. Coronary MR angiography has not yet been established in clinical routine except for the assessment of anomalous origin of coronary arteries. Coronary CT angiography for the assessment of severity of coronary stenosis may be limited by coronary calcium, but lwaki et al. now showed that image quality of MR angiography can also be affected by calcifications. ${ }^{34} \mathrm{~A}$ costeffectiveness study presented by Gosh Dastidar et al. suggested a potential cost saving of $£ 285$ per patient using stress imaging (stress echocardiography or stress (MR) compared with fractional flow reserve guided approaches to manage primary $\mathrm{PCl}$ patients with ST elevation myocardial infarction and additional non-culprit lesions. ${ }^{35}$

\section{Congenital heart disease}

The congenital heart programme at EuroEcho-Imaging 2013 benefited from a strong scientific programme and a large number of abstracts covering the full range of our specialty from foetal 
echocardiography through childhood to adult congenital heart disease. There was strong representation from all imaging sections, often within the one abstract. The RV was a pre-eminent subject and for this reason will be covered by this highlight report. In new borns with hypoplastic left heart syndrome (HLHS), Bellsham-Revell et al. used speckle tracking echo (STE) to measure tricuspid annular descent (TAD) as a measure of RV systemic ventricular function after Norwood 1 palliation. ${ }^{36}$ There was no significant difference in 30-day mortality according to LV morphology, ascending aorta size or TAD\%. A lower TAD\% was seen in those with subjectively impaired RV function (17.7\% vs. $13.6 \%, P=0.001)$ and more than mild TR was a risk factor for death $(P=0.002)$.

Menting et al. investigated the differences in RV deformation in adult congenital patients $>30$ years after repair of tetralogy of Fallot (ToF) or atrial septal defect (ASD). ${ }^{37} \mathrm{RV}$ lateral wall global strain and the strain of all three RV segments separately were significantly lower in ToF and ASD patients than in healthy controls. Strain rate of the RV apical segment was lower in ToF and ASD patients than in controls $(P<0.001, P=0.03)$. LV global strain was reduced in ToF patients $(P<0.001)$ but not in ASD. Finally, RV global strain was positively correlated with LV global strain in both patients groups, which suggests ventricular-ventricular interaction.

Assessment of systemic RV function is key in the follow-up of patients after the atrial switch procedure for transposition of the great arteries (TGA). Many centres consider cardiac CMR as the gold standard for RV assessment. Lypcynska et al. used 2D speckle tracking echo to assess of RV function in this patient group and reported a cut-off strain value to differentiate normal and sub-normal RV systolic function. ${ }^{38}$ They examined 40 adult patients (mean age $25.6 \pm 5$ years; 33 after Senning, 7 after Mustard operation) with $77 \%$ NYHA class I, 20\% in NHYA class II, and 3\% in NHYA class III. There was a linear correlation between GLS and CMR RV ejection fraction values $(r=0.4, P=0.01)$ and between $\mathrm{RV}$ fractional area change and CMR RV ejection fraction ( $r=0.41, P=0.008)$. There was no correlation between RV $s^{\prime}$, TAPSE or RV $d P / d T$ and CMR $\mathrm{RV}$ ejection fraction. Importantly, only 1 out of 19 patients with GLS $<-14.2 \%$ had CMR RV ejection fraction $<45 \%$. A GLS cut-off value of $-14.2 \%$ predicted the CMR RV ejection fraction of $\geq 45 \%$ with $90 \%$ specificity and $83 \%$ sensitivity and a positive predictive of $96 \%$.

In children following the arterial switch operation (ASO) for TGA, Marinov et al. reported that up to $40 \%$ of such children might have perfusion defects on scintigraphy undetected on angiography. ${ }^{39}$ Using GLS they demonstrated a significant difference for both the $\mathrm{RV}$ and LV in this patient group compared with controls. Furthermore, GLS was able to detect abnormality even in those with a normal ejection fraction or TAPSE.

New techniques also featured prominently in the CHD sessions. Two groups compared RV volumes obtained by knowledge-based reconstruction (KBR). Grosse-Wortmann et al. applied KBR to $C M R$ datasets and compared with RV volumes obtained by conventional slice plane summation. ${ }^{40}$ In two separate studies from the same group, Laser et al. ${ }^{41}$ and Horst et al. ${ }^{42}$ aimed to validate KBR volumes obtained from CMR and 3D echo datasets against the gold standard of CMR disc summation. In all three papers, there was good agreement in the volumes, intra- and inter-observer variability, coupled with a short processing time. They suggest that KBR may reduce the time to estimate $\mathrm{RV}$ volume and minimize the need for $\mathrm{CMR}$ in this patient group.

\section{Nuclear cardiology and cardiac computed tomography}

Saraste et al. examined whether short-term spinal cord stimulation (SCS) therapy improves myocardial flow reserve (MFR) and reduces dobutamine-induced myocardial wall motion abnormalities in patients with myocardial ischaemia. ${ }^{43}$ MFR was assessed by positron emission tomography $(\mathrm{PET})$ and $\left[{ }^{15} \mathrm{O}\right] \mathrm{H}_{2} \mathrm{O}$ during rest and adenosine stress. The study showed that short-term SCS treatment improved myocardial flow reserve and reduced dobutamine-induced wall motion abnormalities in patients with ischaemic heart disease and severe angina pectoris, indicating that the treatment had anti-ischaemic effects. Kisko et al. evaluated retrospectively the prognostic value of gated SPECT (single photon emission computed tomography) MPI (myocardial perfusion imaging) in 170 patients with non-stenotic coronary lesions (NSCL) in coronary angiography. ${ }^{44}$ Over a 2-year follow-up, $\sim 11 \%$ of the patients with abnormal SPECT MPI had major coronary events when compared with $3.2 \%$ in those with normal SPECT MPI $(P<0.01)$. The authors recommend gated SPECT MPI in all patients with NSCL. Flori et al. validated Thallium-201 stress-rest ECG-gated SPECT, using a new cadmiumzinc-telluride camera, vs. usual echocardiographic parameters for LV global and regional function analysis. ${ }^{45}$ Correlation between the two methods was good for end-diastolic volume, end-systolic volume, and LV ejection fraction. Correlation was also good regarding regional function. Sunbul et al. using PET evaluated the association of right to LV fluoro-D-glucose uptake ratio (RV/LV FDG-PET) with RV function in 35 patients with chronic thromboembolic pulmonary hypertension. ${ }^{46}$ TAPSE was used as a measure of RV function. It was found that patients with higher RV/LV FDG PET had lower TAPSE, and therefore decreased RV function. Andreini et al. assessed the diagnostic performance of multidetector computed tomography coronary angiography (MDCT-CA) for coronary artery evaluation in 325 patients, who also underwent invasive coronary angiography before TAVI. ${ }^{47}$ MDCT-CA was performed using the same acquisition and dataset employed to standard MDCT aortic annulus evaluation. MDCT-CA showed high sensitivity, specificity, positive predictive value, negative predictive value, and accuracy for detecting $\geq 50 \%$ coronary artery stenosis. The authors recommend routine use of MDCT-CA to rule out significant coronary artery stenosis in patients referred for TAVI. Dobson et al. assessed the incidence of normal, mild ( $<50 \%$ stenosis), and significant $(>50 \%$ stenosis) coronary artery disease (CAD) on computed tomography coronary angiography (CTCA) in a cohort of 148 patients to establish the predictive value of single vs. all three risk factors (diabetes, smoking, hyperlipidaemia). ${ }^{48}$ They also assessed the incidence of CAD based on ethnicity, sex, and typicality of the chest pain. They showed that patients with three risk factors had a much higher incidence of CAD than patients with a single risk factor. The authors pointed out that NICE guidelines need clarification on whether they intended 'high risk' to mean a single risk factor or three risk factors. In addition, typicality of the chest pain was a good predictor of CAD. Cai et al. retrospectively reviewed 213 patients with a moderate or high risk 
of CAD based on NICE guidelines CAD score. ${ }^{49}$ CTCA was performed in 107 , DSE in 67 , and myocardial perfusion scintigraphy in 39 patients. Functional tests led to similar percentage of revasularizations compared with CTCA. The authors concluded that CTCA should be considered as an effective alternative to functional tests in patients with higher pre-test probability of CAD, in hospitals with limited access to functional tests. Barreiro Perez et al. demonstrated that a high percentage of inappropriate indications (according to appropriate use guidelines) for CTCA were noted in a sample of 246 patients, who underwent 64-slice CTCA between 2006 and $2012 .^{50}$ CAD was ruled out significantly more when the indication was appropriate. Patients with inappropriate indication had a greater number of events in the follow-up. Mushtaq et al. investigated the long-term prognostic value of MDCT-CA in a large population of 698 coronary artery bypass graft (CABG) patients and mean followup of $73.5 \pm 14$ months. ${ }^{51}$ Patients were classified by unprotected coronary territory (UCTs) or the coronary artery protection score (CAPS). It was shown that MDCT-CA appears to be a promising tool for long-term risk stratification of CABG patients. Particularly, UCTs performed better than CAPS score for assessment of prognosis. Sunman et al. investigated the diagnostic accuracy of dualsource MDCT-CA in elderly patients in comparison to younger patients. ${ }^{52}$ MDCT-CA results were compared with the results of conventional coronary angiography. A total number of 181 patients were examined with MDCT-CA. Among them, 93 patients were older than 65 years of age 88 were younger. The results showed that sensitivity and positive predictive values were slightly lower for older patients compared with younger. Specificity and negative predictive values were high and similar for both groups.

\section{Funding}

S.E.P. was funded by the National Institute for Health Research Cardiovascular Biomedical Research Unit at Barts.

Conflict of interest: S.E.P. received consulting fees from Circle Cardiovascular Imaging Inc., Calgary, Canada.

\section{References}

1. Muraru D, Calore C, Badano LP, Melacini C, Mihaila S, Peluso D et al. Left ventricular outflow tract planimetry by 3D echocardiography predicts obstruction and heart failure symptoms in hypertrophic cardiomyopathy. Eur Heart J Cardiovasc Imaging 2013;14(Suppl. 2):ii145-72.

2. Huttin O, Voilliot D, Frikha Z, Aliot E, Venner C, Juilliere $Y$ et al. Two dimensional speckle-tracking echocardiography in left ventricular non compaction: reappraisal of global and regional myocardial function. Eur Heart J Cardiovasc Imaging 2013; 14(Suppl. 2):ii176-204.

3. Mansencal N, Arslan M, El Mahmoud El, Pilliere R, Dubourg O. Relationship between recovery and strain pattern by two-dimensional speckle tracking echocardiography: comparison between tako-tsubo cardiomyopathy and acute myocardial infarction. Eur Heart J Cardiovasc Imaging 2013;14(Suppl. 2):ii176-204.

4. Roussin I, Drakopoulou M, Bhattacharyya S, Simpkin V, Sharma R, Rosen S et al. Late cardiac effects of chemotherapy treatment: the role of ejection fraction and more subtle indices of cardiac dysfunction. Eur Heart J Cardiovasc Imaging 2013;14(Suppl. 2):ii145-72.

5. Hasselberg NE, Hauggaa KH, Brunet A, Kongsgaard E, Donal E, Edvardsen T. Global longitudinal strain predicts subsequent ventricular arrhythmia in patients receiving cardiac resynchronization therapy. Eur Heart J Cardiovasc Imaging 2013;14(Suppl. 2):ii20-48.

6. Hasselberg NE, Hauggaa KH, Brunet A, Kongsgaard E, Donal E, Edvardsen T. Mechanical dyssynchrony is a marker for ventricular arrhythmia in patients with cardiac resynchronization therapy. Eur Heart J Cardiovasc Imaging 2013;14(Suppl. 2):ii64-6.

7. Bernard-Brunet A, Donal E, Reynaud A, Schnell F, DaubertJC, Leclercq C et al. New insights into left ventricular mechanics with the analysis of longitudinal strain integrals on heart failure patients. Eur Heart J Cardiovasc Imaging 2013;14(Suppl. 2):ii145-72.
8. Bandera F, Pellegrino M, Generati G, Alfonzetti E, Donghi V, Castelvecchio S et al. Assessing right ventricular functional response during incremental exercise in heart failure: insights on mitral regurgitation and ventilatory efficiency. Eur Heart J Cardiovasc Imaging 2013;14(Suppl. 2):ii145-72.

9. Bandera F, Guazzi M, Arena R, Corra U, Ghio S, Forfia Pet al. Powerful prognostic and clinical insights combining TAPSE and PASP in heart failure: correlation and additive value with cardiopulmonary exercise response. Eur Heart J Cardiovasc Imaging 2013; 14(Suppl. 2):ii99-ii126.

10. Tsang W, Salgo IS, Gajjar M, Abduch MC, Freed BH, Weinert L et al. A new fourparameter echocardiographic score to predict mortality with aortic stenosis. Eur Heart J Cardiovasc Imaging 2013;14(Suppl. 2):ii16.

11. Carstensen HG, Larsen LH, Hassager C, Kofoed KF, Kristensen CB, Jensen JS et al. Global and especially regional longitudinal function is associated with severity in moderate-severe aortic stenosis. Eur Heart J Cardiovasc Imaging 2013;14(Suppl. 2): ii31.

12. Dulgheru R, Magne J, Kou S, Machado S, Henri C, Voilliot D et al. Left ventricular basal longitudinal systolic dysfunction is a determinant of maximal exercise capacity in patients with asymptomatic aortic stenosis. Eur Heart J Cardiovasc Imaging 2013; 14(Suppl. 2):ii31.

13. Federspiel M, Oger E, Fournet M, Daudin M, Thebault C, Donal E. Value of exercise stress echocardiography in elderly asymptomatic patients having a severe aortic stenosis. Eur Heart J Cardiovasc Imaging 2013;14(Suppl. 2):ii17.

14. Fabris E, Morosin M, Moretti M, Pinamonti B, Merlo M, Barbati G et al. Low-gradient aortic stenosis with preserved left ventricular ejection fraction: prognostic stratification in operated and non-operated patients. Eur Heart J Cardiovasc Imaging 2013; 14(Suppl. 2):ii16.

15. Sato K, Seo Y, Ishizu Y, Takeuchi M, Izumo M, Suzuki K et al. Prognostic value of longitudinal strain in patients with low-flow, low-gradient severe aortic stenosis despite preserved ejection fraction. Eur Heart J Cardiovasc Imaging 2013;14(Suppl. 2):ii31.

16. Knebel F, Spethmann S, Baldenhofer G, Sanad W, StangI V, Laule Met al. Recovery of left ventricular systolic function in different entities of aortic stenosis 12 months after TAVI: A 2 SD speckle tracking study. Eur Heart J Cardiovasc Imaging 2013;14(Suppl. 2): ii31.

17. Cameli M, Lisi M, Righini FM, Di Tommaso C, Curci V, Cameli Pet al. Left atrial strain for prognosis prediction in patients with moderate mitral valve regurgitation. Eur Heart J Cardiovasc Imaging 2013;14(Suppl. 2):ii18.

18. Kozdag G, Ertas G, Emre E, Akay Y, Karauzum K, Yilmaz I et al. Moderate to severe tricuspid regurgitation is an independent predictor for early readmission in patients with chronic heart failure. Eur Heart J Cardiovasc Imaging 2013;14(Suppl. 2):ii18.

19. Brugger N, De Marchi S, Steck H, Zumstein D, Seiler C. Impact of the nyquist limit on mitral regurgitant flow rate as measured by 3-dimensional proximal isovelocity surface area. Eur Heart J Cardiovasc Imaging 2013;14(Suppl. 2):ii3.

20. Condemi F, Bapat V, Gianstefani S, Catibog N, Monaghan MJ. Validation of 2D \& 3D TEE measurement of prosthetic valve dimensions - implications for percutaneous valve in valve therapy. Eur Heart J Cardiovasc Imaging 2013;14(Suppl. 2):ii138.

21. Veronesi F, Addetia K, Corsi C, Lamberti C, Lang RM, Mor-Avi V. Estimates of mitral-aortic angle measurement errors in 2D compared to 3D echocardiography. Eur Heart J Cardiovasc Imaging 2013;14(Suppl. 2):ii77.

22. Brugger N, Huerzeler M, Wustmann K, Wahl A, Steck H, Seiler C. Evaluation of chronic mitral regurgitation by 3-D color Doppler echocardiography vs cardiac magnetic resonance imaging as the reference method. Eur Heart J Cardiovasc Imaging 2013;14(Suppl. 2):ii164.

23. Muraru D, Piasentini E, Mihaila S, Padayattil Jose' S, Peluso D, Ucci L et al. Reference ranges for left ventricular geometry and function by $3 \mathrm{D}$ echocardiography using a vendor-independent software for quantitative analysis. Eur Heart J Cardiovasc Imaging 2013;14(Suppl. 2):ii205.

24. Shivalkar B, Belkova P, Wouters K, Van De Heyning C, De Maeyer C et al. Dobutamine stress echocardiography: qualitative and quantitative analysis beyond $2 \mathrm{D}$. Eur Heart J Cardiovasc Imaging 2013;14(Suppl. 2):ii206.

25. Carminati MC, Piazzese C, Tsang W, Lang RM, Caiani EG. Automated fusion of 3D TEE descending aortic datasets for improved examination and quantification of atheromas burden. Eur Heart J Cardiovasc Imaging 2013;14(Suppl. 2):ii60.

26. Debonnaire P, Al Amri I, Leong DP, Joyce E, Katsanos S, Kamperidis V et al. Leaflet remodeling in functional mitral valve disease: characteristics, determinants and relation to regurgitation severity. Eur Heart J Cardiovasc Imaging 2013;14(Suppl. 2): ii155.

27. Grapsa J, Cabrita IZ, Durighel G, O'regan D, Dawson D, Nihoyannopoulos P. Right ventricular remodeling in idiopathic pulmonary arterial hypertension assessed with 3D echocardiography and CMR: assessment of the learning curve of both modalities. Eur Heart J Cardiovasc Imaging 2013;14(Suppl. 2):ii209.

28. Orwat S, Diller GP, Radke RM, Bauerschmitz PB, Schmidt R, Baumgartner H. Myocardial strain as measured by CMR relates to functional status, objective exercise capacity and neurohormonal levels in adults with congenitally corrected transposition of the great arteries. Eur Heart J Cardiovasc Imaging 2013;14(Suppl. 2):ii29-30. 
29. Franzoso F, Voser EM, Wohlmut C, Kellenberger CJ, Valsangiacomo Buechel E. Atrial versus arterial switch repair for transposition of great arteries. CMR evaluation of left and right atrial function; a comparative study. Eur Heart J Cardiovasc Imaging 2013;14(Suppl. 2):ii226.

30. Axelsson A, Langhoff L, Jensen MK, Vejlstrup N, Iversen K, Bundgaard H. Comparison of 2D Echocardiographic methods with MRI in the assessment of left atrial volume in hypertrophic cardiomyopathy. Eur Heart J Cardiovasc Imaging 2013; 14(Suppl. 2):ii100.

31. Halmai L, Nemes A, Kardos A, Neubauer S. Can 3D-Speckle Tracking analysis be useful for identifying patients with acute myocarditis? A comparison with cMRI finding. Eur Heart J Cardiovasc Imaging 2013;14(Suppl. 2):ii43.

32. Marques JS, Almeida AMG, Menezes M, Silva GL, Placido R, Amaro C et al. Relationship of CMR myocardial late-gadolinium enhancement and cardiac biomarkers in patients with acute myocarditis: a prospective study. Eur Heart J Cardiovasc Imaging 2013;14(Suppl. 2):ii114.

33. Poyhonen P, Kivisto S, Holmstrom M, Hanninen H. Late gadolinium enhancement and segmental wall motion abnormalities on CMR predict adverse outcome in suspected non-ischemic cardiomyopathy. Eur Heart) Cardiovasc Imaging 2013;14(Suppl. 2):ii80.

34. Iwaki T. The influence of the arc and length of calcification to image quality of coronary MRA. Eur Heart J Cardiovasc Imaging 2013;14(Suppl. 2):ii144.

35. Ghosh Dastidar A, Augustine D, Cengarle M, Mcalindon E, Bucciarelli-Ducci C, Nightingale A. Stress imaging guided strategy vs. fractional flow reserve guided approach in patients with non-culprit lesion at primary percutaneous coronary intervention: comparative effectiveness and cost. Eur Heart J Cardiovasc Imaging 2013; 14(Suppl. 2):ii106.

36. Bellsham-Revell H, Bell A, Miller OI, Simpson JM. Echocardiographic risk factors for 30 day mortality after the Norwood procedure for hypoplastic left heart syndrome. Eur Heart J Cardiovasc Imaging 2013;14(Suppl. 2):ii20-48.

37. Menting ME, van den Bosch AE, McGhie JS, Eindhoven JA, Cuypers JAAE, Witsenburg $M$ et al. Right Ventricular Myocardial Deformation: Differences between corrected Tetralogy of Fallot and Atrial Septal Defect. Eur HeartJ Cardiovasc Imaging 2013;14(Suppl. 2):ii176-204.

38. Lipcynska M, Symanski P, Klisiewicz A, Mazurkiewicz L, Hoffman P. Do we need routine systemic RV functions assessed by $\mathrm{CMRI}$ in all patients with complete TGA following the atrial switch procedure? Eur Heart J Cardiovasc Imaging 2013; 14(Suppl. 2):ii20-48.

39. Marinov R, Hristova K, Georgiev S, Kaneva A, Katova T, Pilossoff V. Left ventricle/ right ventricle interaction in youth population with D-TGA. a speckle tracking study. Eur Heart J Cardiovasc Imaging 2013;14(Suppl. 2):ii29-30.

40. Grosse-Wortmann L, Nyns E, Yoo S-J, Dragulescu A. KBR for MRI RV volumetry after ASO for TGA. Eur Heart J Cardiovasc Imaging 2013;14(Suppl. 2):ii29-30.
41. Laser KT, HorstJP, Kelter-Klöpping A, Barth P, Haas N, Kececioglu D et al. Multimodality quantification software for ventricular volumes - Is landmark-based volumetry the new answer for the right ventricle? Eur Heart) Cardiovasc Imaging 2013;14(Suppl. 2):ii45-172.

42. HorstJ-P, Kelter-Klöpping A, Koerperich H, Barth P, Haas N, Kececioglu D et al. Right ventricular RT3DE using Angelo Software in CHD - Is MRI still gold standard? Eur Heart J Cardiovasc Imaging 2013;14(Suppl. 2):ii45-172.

43. Saraste A, Luotolahti M, Varis A, Vasankari T, Tunturi S, Taittonen M et al. Effect of spinal cord stimulation on longitudinal myocardial strain in patients with severe angina pectoris. Eur Heart J Cardiovasc Imaging 2013;14(Suppl. 2):ii106.

44. Kisko A, Vereb M. Myocardial perfusion imaging in non-stenotic coronary lesions: does it make sense?. Eur Heart J Cardiovasc Imaging 2013;14(Suppl. 2):ii53.

45. Flori M, Valette F, Guijarro D, Pallardy A, Le Tourneau T, Kraeber-Bodere F et al. Absolute quantification of left ventricular global and regional function during myocardial perfusion imaging with new CZT camera: initial validation versus echocardiography. Eur Heart J Cardiovasc Imaging 2013;14(Suppl. 2):ii170.

46. Sunbul M, Kivrak T, Oguz M, Ozguven S, Gungor S, Dede F et al. Evaluation of association between fluoro-d-glucose positron emission tomography uptake with right ventricular functions in patients with chronic thromboembolic pulmonary hypertension. Eur Heart J Cardiovasc Imaging 2013;14(Suppl. 2):ii93.

47. Andreini D, Pontone G, Mushtaq S, Bertella E, Conte E, Baggiano A et al. Diagnostic accuracy of multidetector computed tomography coronary angiography. Eur Heart J Cardiovasc Imaging 2013;14(Suppl. 2):ii54.

48. Dobson P, Cai P, Leung P, Marshall K, Albarjas M, Rogers T et al. Predicting the incidence of CAD in CT coronary angiography based on the number of cardiac risk factors, ethnicity, sex and typicality of chest pain. Eur Heart J Cardiovasc Imaging 2013;14(Suppl. 2):ii53.

49. Cai A, Dobson P, Leung P, Marshall K, Albarjas M, Rogers T et al. A comparison between $\mathrm{CT}$ coronary angiography and functional tests, in patients with stable chest pain and moderate to high predicted risk of coronary artery disease. Eur Heart J Cardiovasc Imaging 2013;14(Suppl. 2):ii54.

50. Barreiro Perez M, Martin Fernandez M, Cimadevilla Fernandez OC, Renilla Gonzalez A, Diaz Pelaez E, Rozado Castano J. Analysis of coronary CT angiography indications in symptomatic patients according 2006 and 2010 appropriate use Guidelines. Eur Heart J Cardiovasc Imaging 2013;14(Suppl. 2):ii53.

51. Mushtaq S, Andreini D, Pontone G, Bertella E, Conte E, Baggiano A et al. Prognostic value of $C T$ angiography in coronary bypass patients: a 73 months follow-up study. Eur Heart J Cardiovasc Imaging 2013;14(Suppl. 2):ii202.

52. Sunman H, Yorgun H, Sahiner L, Kaya B, Hazirolan T, Ozer N et al. Comparison of multidetector computed tomography coronary angiography with conventional angiography in elderly and younger patients. Eur Heart J Cardiovasc Imaging 2013; 14(Suppl. 2):ii144. 